\title{
Regulating the biosynthesis of pyridoxal 5'-phosphate with riboswitch to enhance L-DOPA production by Escherichia coli whole-cell biotransformation
}

\author{
Hongmei $\operatorname{Han}^{1}$, Bingbing Xu${ }^{1}$, Weizhu Zeng ${ }^{2}$, and Jingwen Zhou ${ }^{2}$ \\ ${ }^{1}$ Jiangnan University \\ ${ }^{2}$ Jiangnan Univ.
}

May 5, 2020

\begin{abstract}
Pyridoxal 5'-phosphate (PLP) is an essential cofactor that participates in 4\% enzymatic activities cataloged by the Enzyme Commission. The intracellular level of PLP is usually lower than that demanded in industrial catalysis. To realize the self-supply of PLP cofactor in whole-cell biotransformation, the de novo ribose 5-phosphate (R5P)-dependent PLP synthesis pathway was constructed. The pdxST genes from Bacillus subtilis 168 were introduced into the tyrosine phenol-lyase (TPL)-overexpressing Escherichia coli BL21(DE3) strain. TPL and PdxST were co-expressed with a double-promoter or a compatible double-plasmid system. The 3,4-dihydroxyphenylacetate-L-alanine (L-DOPA) titer did not increase with the increase in the intracellular PLP concentration in these strains with TPL and PdxST co-expression. Therefore, it is necessary to optimize the intracellular PLP metabolism level so as to achieve a higher L-DOPA titer and avoid the formation of L-DOPA-PLP cyclic adducts. The thi riboswitch binds to PLP and forms a complex such that the ribosome cannot have access to the SD sequence. Therefore, this metabolite-sensing regulation system was applied to reduce the translation of mRNA. Riboswitch was introduced into pET-TPL-pdxST-2 to downregulate the expression of PdxST and biosynthesis of PLP at the translation level by sequestering the ribosome-binding site. As a result, the titer and productivity of L-DOPA using the strain BL21-TPLST-Ribo1 improved to $69.8 \mathrm{~g} / \mathrm{L}$ and $13.96 \mathrm{~g} / \mathrm{L} / \mathrm{h}$, respectively, with a catechol conversion of $95.9 \%$ and intracellular PLP accumulation of $24.8 \mu \mathrm{M}$.
\end{abstract}

\section{Hosted file}

HanHM-PLP-20200107-ZhouJW. doc available at https ://authorea.com/users/291539/articles/418844regulating-the-biosynthesis-of-pyridoxal-5-phosphate-with-riboswitch-to-enhance-1-dopaproduction-by-escherichia-coli-whole-cell-biotransformation
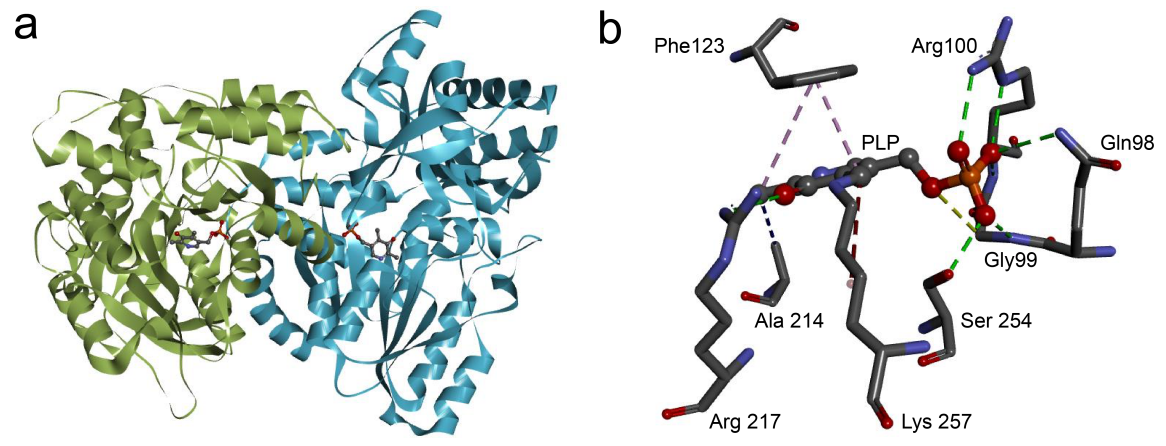
pET-28-TPL(E313M)

T7 $\longrightarrow$ SD TPL T7 terminator

pCDFDuet-pdxST'

Trc $\longrightarrow \mathrm{SD} p d x S$ SD $p d x T$ T7 terminator

pCDFDuet-pdxST

$\mathrm{T} 7 \mathrm{SD} p d x \mathrm{SD} p d x T$ T7 terminator

pETDuet-pdxST

$\mathrm{T} 7 \mathrm{SD} p d x \mathrm{SD} p d x T$ T7 terminator

pRSFDuet-pdxST

T7Г SD $p d x S$ SD $p d x T$ T7 terminator

pET-TPL-pdxST-1

T7 $\longrightarrow$ SD TPL Trc $\longrightarrow$ SD pdxS SD pdxT T7 terminator pET-TPL-pdxST-2
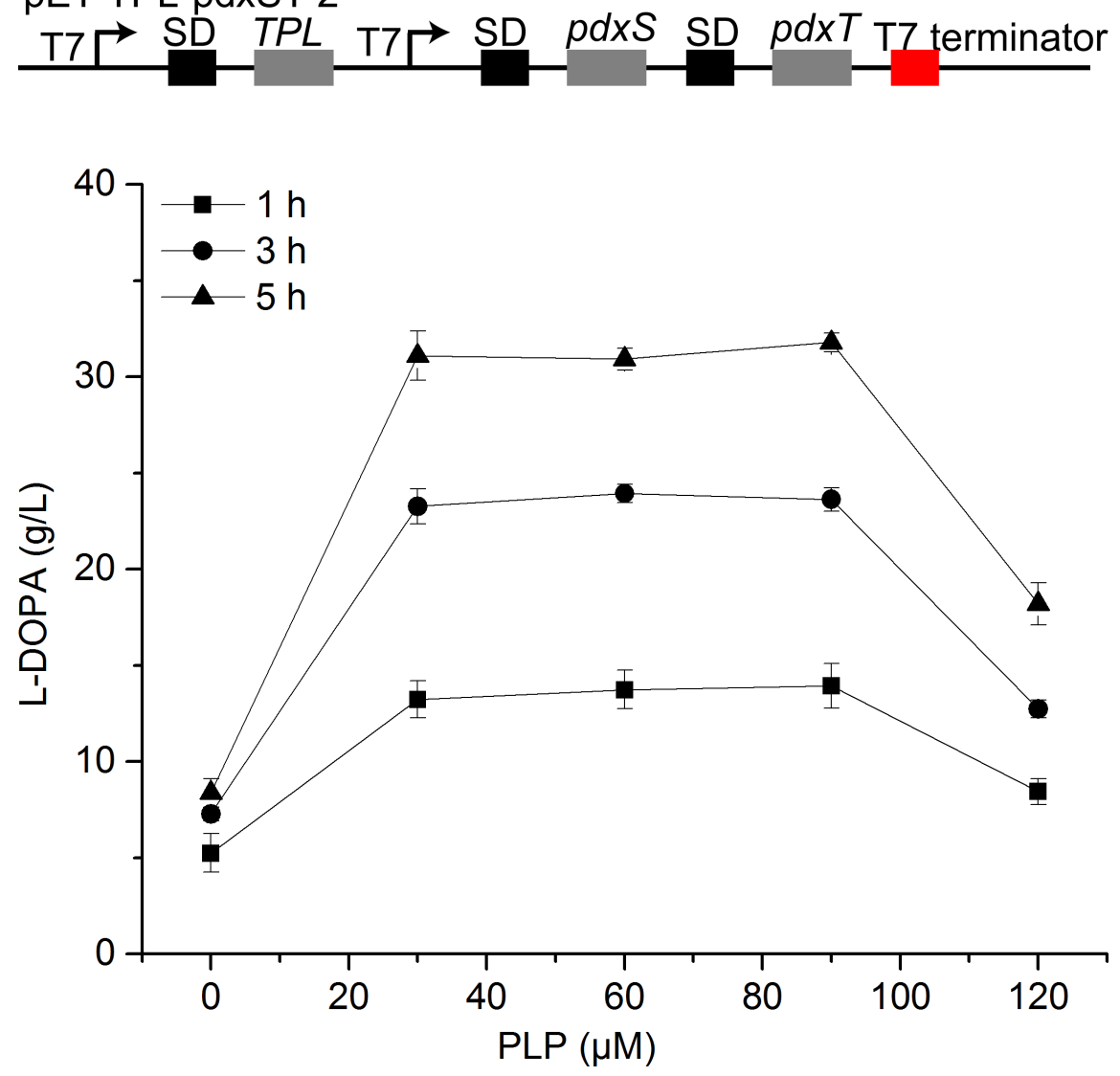

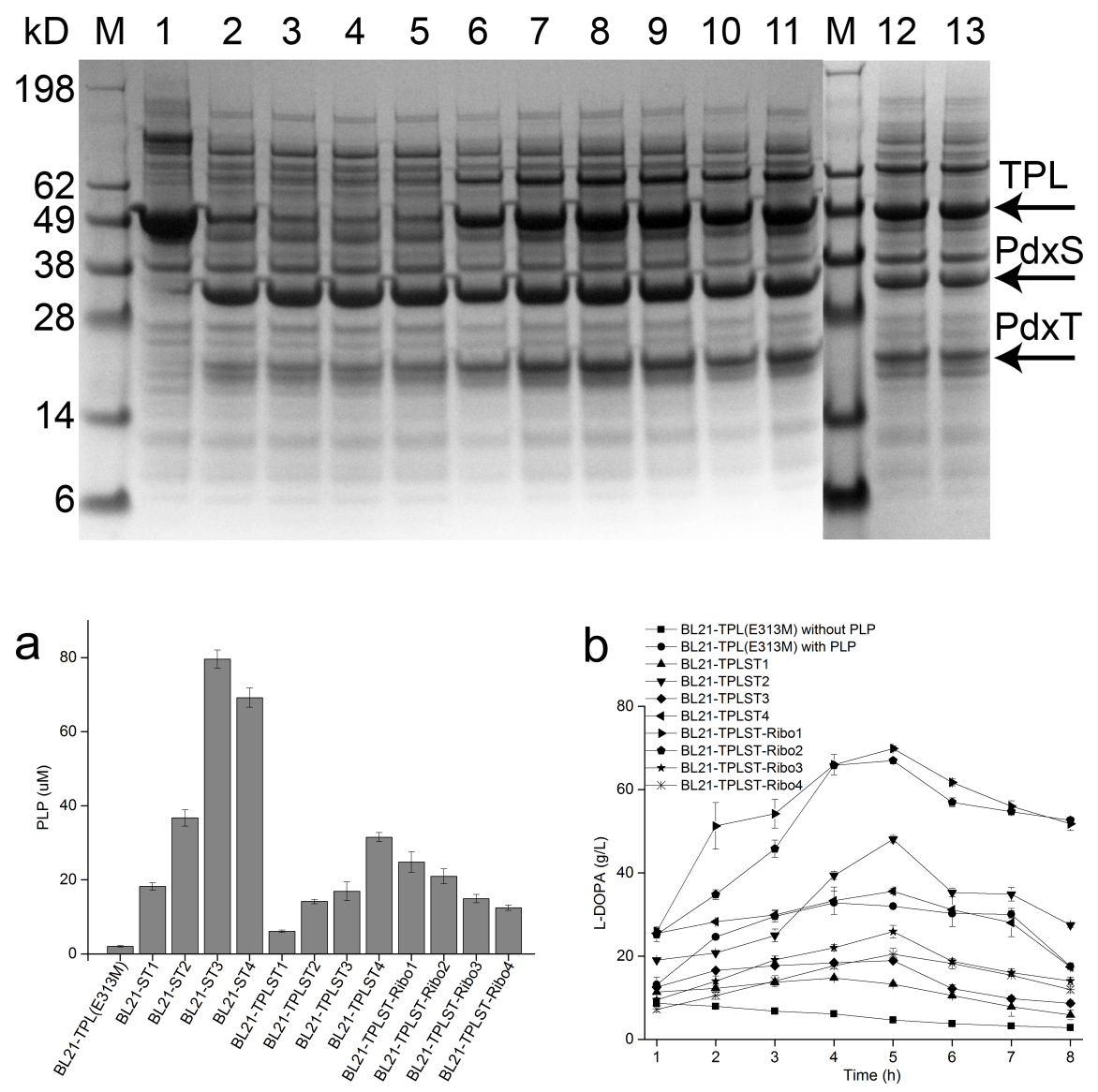

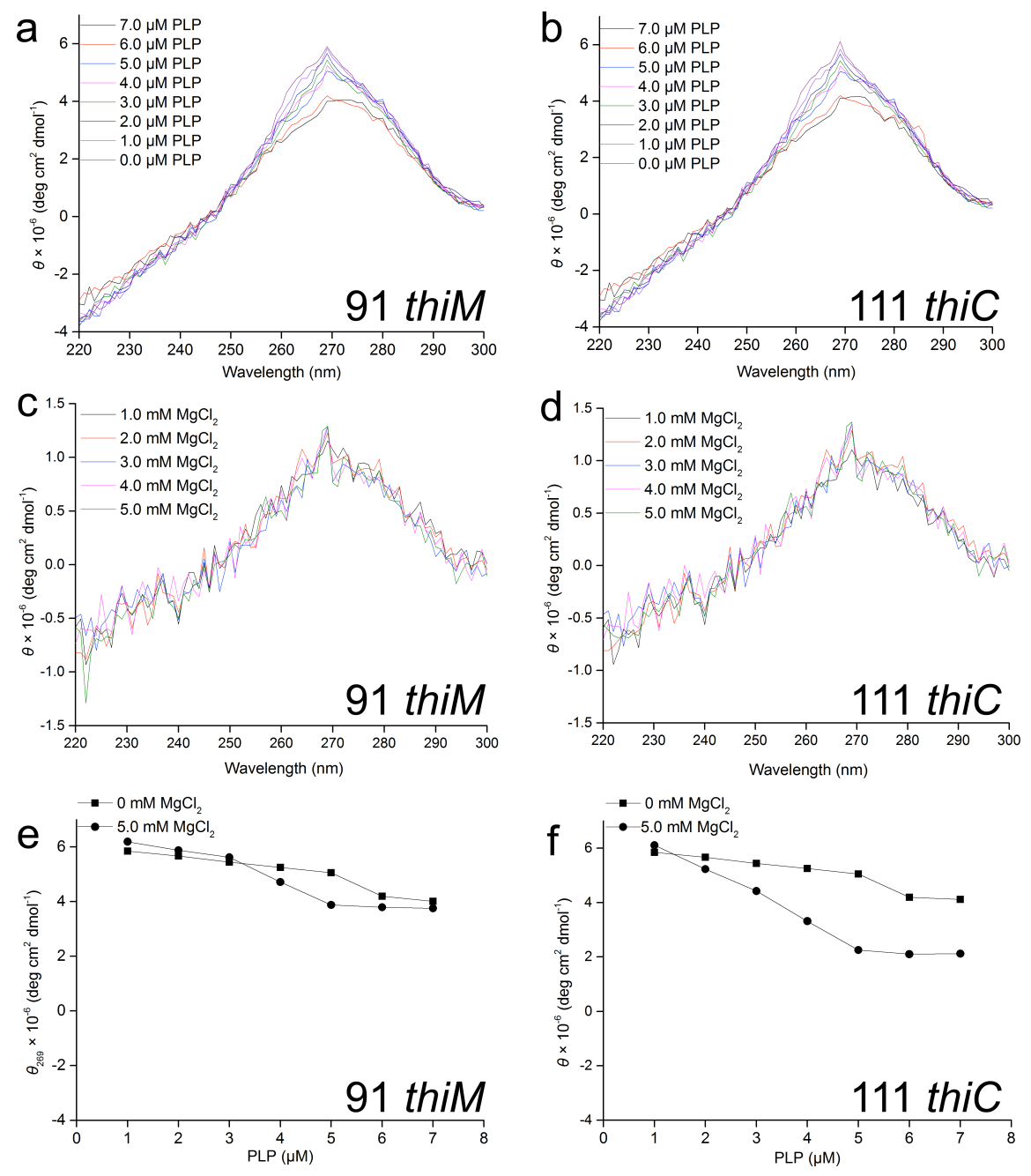

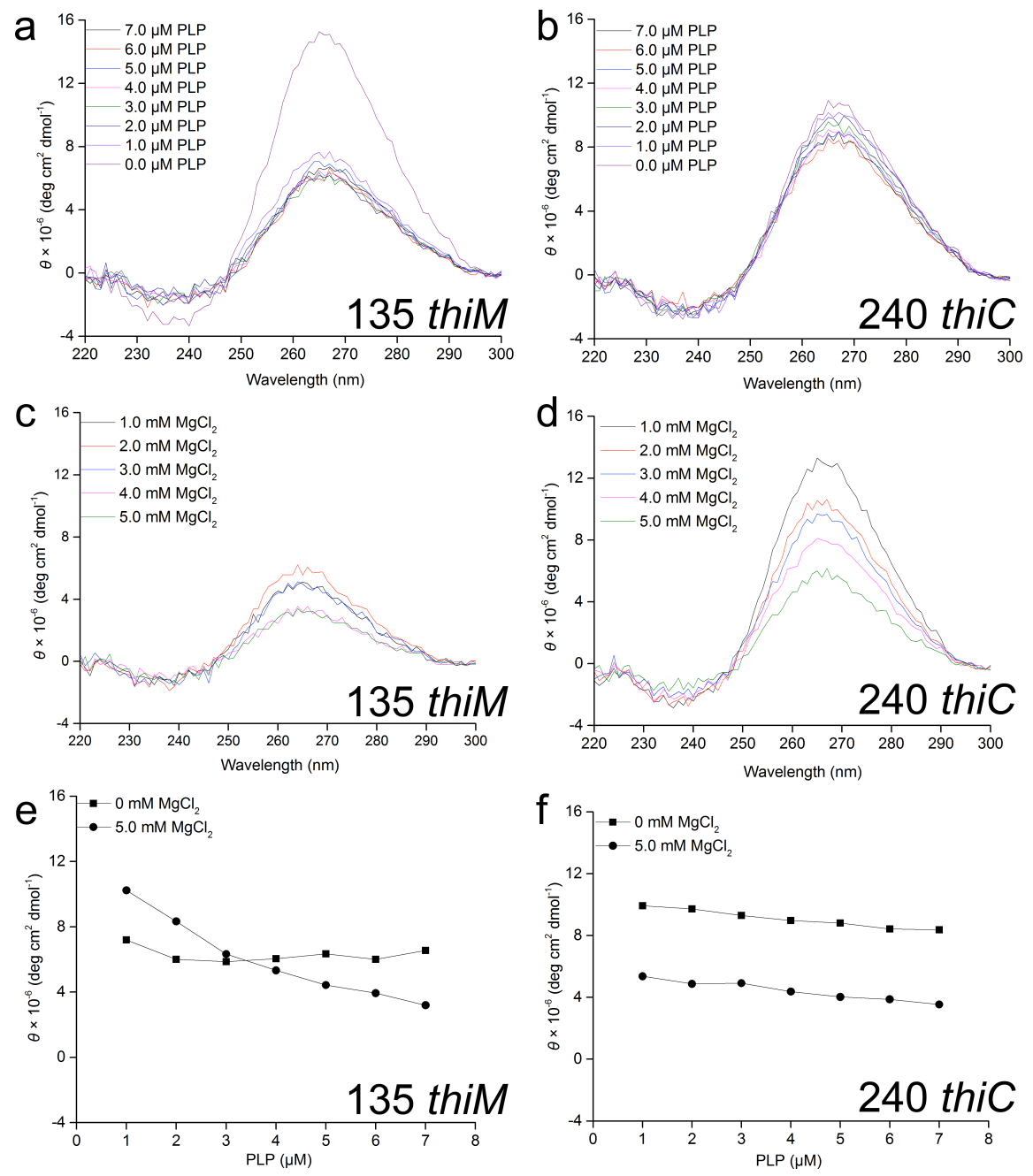


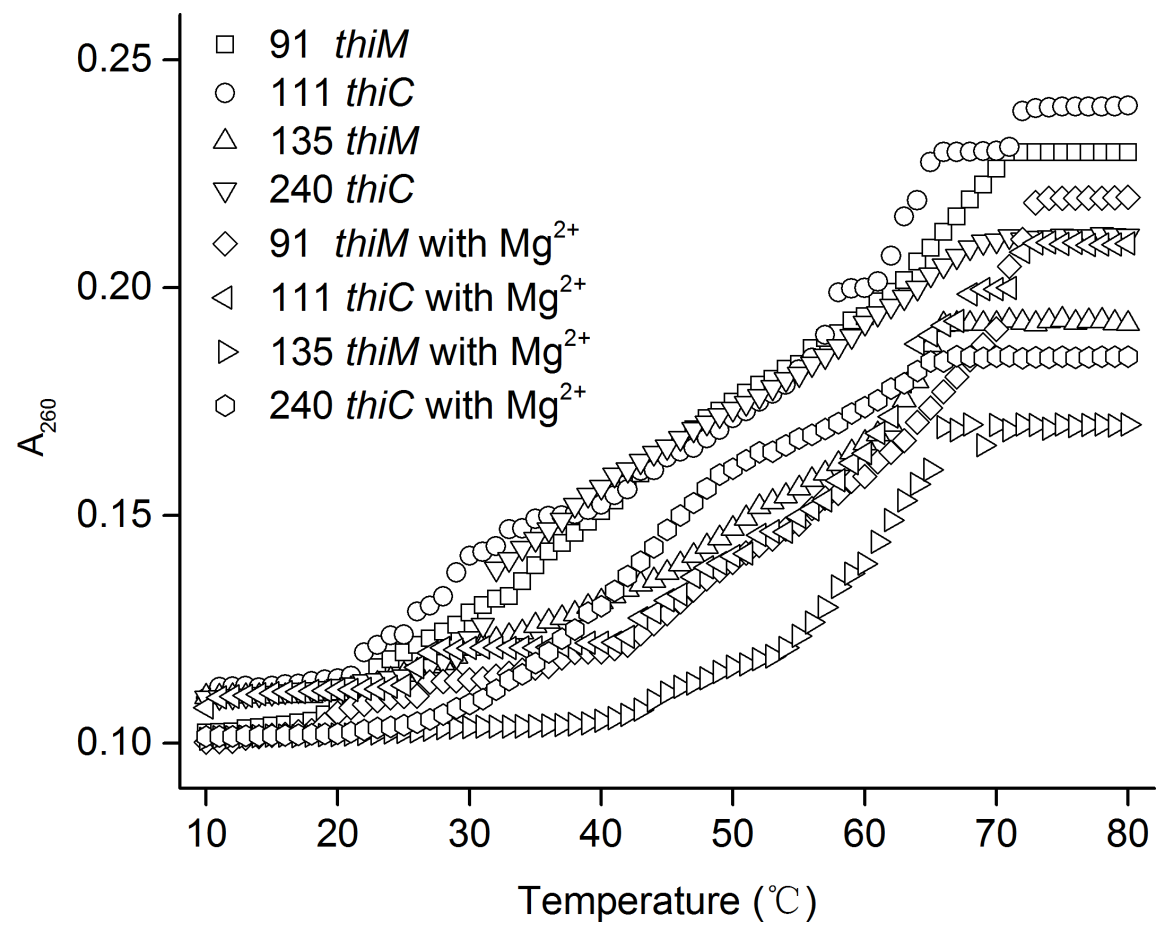

